\title{
Une vision allemande du 18 Brumaire
}

Les résultats de ma mission à Paris dans le mois de brumaire an VIII, de Joseph von Görres

\section{Marita Gilli}

\section{(e) OpenEdition \\ 1 Journals}

Édition électronique

URL : https://journals.openedition.org/ahrf/300

DOI : $10.4000 /$ ahrf.300

ISSN : 1952-403X

Éditeur :

Armand Colin, Société des études robespierristes

Édition imprimée

Date de publication : 1 décembre 1999

Pagination : 747-754

ISSN : 0003-4436

\section{Référence électronique}

Marita Gilli, « Une vision allemande du 18 Brumaire », Annales historiques de la Révolution française [En ligne], 318 | octobre-décembre 1999, mis en ligne le 11 avril 2006, consulté le 24 avril 2022. URL : http://journals.openedition.org/ahrf/300 ; DOl : https://doi.org/10.4000/ahrf.300

Ce document a été généré automatiquement le 24 avril 2022.

Tous droits réservés 


\section{Une vision allemande $\mathrm{du}$ 18 Brumaire}

Les résultats de ma mission à Paris dans le mois de brumaire an VIII, de Joseph von Görres

\section{Marita Gilli}

1 Görres est un des rares penseurs révolutionnaires allemands à être passé à la pratique politique. D'abord enthousiasmé par les événements de Mayence, il s'investit dans la vie politique à Coblence lors de la deuxième phase d'occupation française. Il fonde un journal La feuille rouge ${ }^{1}$ dans le but d'éduquer le peuple allemand et de créer l'esprit républicain. Lors de la reprise de Mayence le 12 nivôse an VI, il annonce la mort du Saint-Empire et déclare la République française seule héritière de toute la rive gauche du Rhin. Auteur de nombreuses professions de foi révolutionnaires, il critique l'Ancien Régime avec une ironie mordante. Mais les réalités de l'administration sous tutelle française pendant le Directoire ne tardent pas à le décevoir la corruption s'installe, le manque de liberté politique est flagrant ${ }^{2}$. Görres dénonce la situation dans son journal qui se voit interdit et doit changer de titre, devenant le Rübezahl. Görres passe même quelques jours en prison, mais continue à exprimer ses critiques. La conclusion d'une adresse au corps législatif est tout à fait révélatrice de son état d'esprit «Citoyens législateurs! Trente millions d'hommes vous ont confié leur salut et réclament les droits que vous avez de faire leur bien-être civil et moral avec confiance, ils jettent les regards vers vous et attendent de votre énergie la destruction totale des bandes qui les ont tenus enchaînés (...). Détruisez les présages des hommes craintifs ou malveillants qui, dans votre victoire, n'entrevoient que le triomphe du parti, ou seulement un changement des rôles et non des choses (...). Que la mort aux frippons, le bannissement à l'arbitraire et la réunion à tous les braves républicains soit votre mot de ralliement, et le nôtre sera, notre vie à la patrie et à la liberté. $»^{3}$

2 Dès avril 1799 Görres était partisan d'envoyer une députation des départements rhénans à Paris afin de solliciter le rattachement officiel des territoires occupés à la France. En effet, la situation s'était considérablement dégradée dans les territoires rhénans et le 30 prairial avec l'entrée de Sieyès au Directoire avait été favorablement 
perçu par les républicains rhénans. D'un autre côté, la situation militaire devenait inquiétante, Mayence risquait d'être reprise et cela avait redoublé l'énergie des républicains. C'est cette situation qui détermine l'envoi de la mission à Paris, mission dont on attend qu'elle améliore la situation interne et qu'elle assure l'existence du pays. Les objectifs qui lui sont assignés sont clairs obtenir du gouvernement français la réunion définitive de la rive gauche du Rhin, présenter la défense de Lakanal qui était en grande difficulté et « dévoiler les intrigues tramées par les ennemis de la République contre la liberté » dans les départements rhénans. Lorsque Görres arrive à Trèves où doivent se rejoindre les délégués des quatre départements, la nouvelle du coup d'État du 18 Brumaire vient d'y parvenir. L'importance de l'événement ne leur échappe pas "Un acte du drame était terminé, un autre commençait " ${ }^{4}$. Bonaparte pouvait aussi bien entrer sur la scène en tant que traitre que pour sauver la France du bord du précipice dans lequel elle risquait de sombrer alors que leurs collègues estiment inutile désormais l'envoi de la délégation à Paris, Görres et le général Eickemeyer, délégué du Mont-Tonnerre, décident de partir ils arrivent à Paris le 30 brumaire.

De cette mission, nous restent deux textes les Résultats de ma mission à Paris et les lettres à sa fiancée Katharina von Lassaulx ${ }^{5}$. Si le compte rendu de la mission présente une analyse politique et raisonnée des événements, les lettres expriment surtout son état d'âme, en particulier le peu de sympathie qu'il éprouve pour les Français, allant jusqu'à déclarer qu'il ne les aime pas. Il se sent exilé dans un monde qui lui est étranger et ressent la nostalgie de sa patrie rhénane, ce qui explique peut-être en partie certaines de ses positions.

4 Ce qui frappe Görres à son arrivée, c'est le peu d'intérêt porté aux nouveaux départements et l'ignorance par rapport à ce qui s'y passe. Il constate que la ruse a succédé aux passions. Par ailleurs son jugement sur Bonaparte se démarque de ce que l'on pensait généralement à ce moment alors qu'une grande partie de l'opinion publique en Allemagne voit en lui le garant d'un retour à l'ordre, Görres redoute aussitôt de " voir les conquêtes de la Révolution tout entière englouties par l'ambition d'un seul homme» (p. 562). D'emblée, il prévoit donc l'avènement d'un régime dictatorial. L'ancienne Constitution a été détruite, la nouvelle est déjà en vigueur et les deux délégués cherchent à connaître le terrain dans lequel ils devront évoluer. Depuis le 18 Brumaire, le gouvernement français s'était fixé comme maxime de mettre fin aux dissensions qui avaient miné l'État. Lakanal est destitué et Görres rédige des mémoires pour les présenter à son successeur Dubois Dubais. Il y explique les différences entre les peuples du Nord réfléchis, modérés et tenant à leurs principes et ceux du Sud, s'enflammant plus facilement, enthousiasmés et violents qu'on ne peut donc gouverner de la même façon. Dubois Dubais est ensuite remplacé par Shee qui, lors d'une visite que les deux délégués lui rendent à Nanterre, les reçoit avec des paroles réconfortantes, disant qu'il s'efforcera de faire le bien dans un pays qu'il connaît déjà.

5 Les deux délégués doivent également déterminer le gouvernement à fixer définitivement le sort de la rive gauche du Rhin. Pour cela ils étudient la nouvelle Constitution afin de voir si elle pourra leur convenir. Görres résume ainsi la question qui se pose «Dans quelle mesure l'humanité peut-elle encore continuer à se réclamer de la Révolution française, étant donné le tournant que cet événement universel a pris depuis le 18 brumaire et quelles exigences nationales particulières sont en contradiction avec les enseignements universels qu'on en tire » (p. 570). Pour répondre à cette question, fondamentale pour lui, il retrace l'histoire de la Révolution française 
dans le but d'expliquer les raisons du coup d'État du 18 Brumaire qui représente pour lui la conséquence directe des événements qui l'ont précédé.

6 On a pensé que le peuple français était capable de réaliser les idéaux de la Révolution, mais l'expérience a montré selon lui qu'il n'en était rien. Les débuts de la Révolution n'ont été qu'affaire de sentiment et de passions. Brossant un tableau de la Révolution française jusque là, il montre qu'un premier schisme s'est révélé entre la majorité de la nation et la minorité de véritables révolutionnaires la masse s'est selon lui vite détournée de la Révolution et est devenue indifférente. Le second schisme a affecté les dirigeants eux-mêmes, opposant les Girondins qu'il qualifie de "théoriciens de la pratique sans sens pratique actif» et les Jacobins qu'il qualifie de "praticiens de la théorie sans esprit spéculatif ordonné » (p. 574). Devant la résistance à l'intérieur et à l'extérieur, le terrorisme est devenu le régulateur de l'action des Jacobins. Cette période de la Révolution a prouvé que le peuple manquait de maturité et que les gouvernants n'ont pas été capables de gouverner. Le Directoire va donner le pouvoir à une " aristocratie organisée de la culture ». Mais l'histoire du Directoire est celle d'une dégradation de la moralité publique, la spéculation et la corruption des mœurs s'étant accrues. C'est ainsi que s'est créé un nouveau schisme entre la majorité nationale et les brigands qui la dirigeaient. Ce troisième schisme se produit avec le coup d'État du 18 fructidor puis un quatrième avec le 30 prairial, révolte de la nation contre la corruption des gouvernants. Mais ce sursaut a également été voué à l'échec en raison du refus du recours à la force.

7 Telle est la situation que trouve Bonaparte en rentrant en France. Dans les pays étrangers, elle est encore pire. Où trouver les moyens de sortir de cet état Les Jacobins ont été rejetés, Sieyès a échoué dans sa tentative de domination par l'esprit et la grandeur de caractère. L'entreprise avait été trop importante pour la nature humaine, trop importante pour celle du peuple français. La Révolution se trouve donc dans l'impasse et il ne lui reste plus qu'à confier le pouvoir à un de ces individus hors pair qui allient la grandeur d'esprit et la force de caractère. Or Bonaparte rentre d'Égypte victorieux il possède toutes les qualités qui permettent de voir en lui un sauveur de la nation, ses victoires ont témoigné de son génie, il a pu montrer sa force de caractère à plusieurs reprises. Il fallait du courage pour prendre la direction de la France dans l'état où elle était, mais il a agi vite, sans hésitations à l'extérieur, la France est de nouveau au faîte de sa puissance, à l'intérieur le calme est revenu.

8 Tel est le sens du 18 Brumaire «Après un combat furieux pendant lequel des milliers d'existences furent sacrifiées au bonheur futur, on se trouva contraint, après avoir été peu à peu chassé de retranchement en retranchement, de se jeter à nouveau dans les bras d'un unique caractère, d'un unique esprit et de supplier celui-ci de vous sauver du gouffre qui venait de s'ouvrir sous vos pieds»(p. 586). Le 18 Brumaire est la conséquence naturelle de ce qui l'a précédé ce n'est pas Sieyès qui a voulu ce coup d'État, mais la nature. La Révolution a manqué son but et il faudra par la suite étudier les erreurs commises afin de poursuivre ce qui a été commencé. Devant le danger, la France a fait ce qu'il est naturel de faire elle a choisi la force qui restait en son sein et a remis son destin entre ses mains. "Par cette démarche, elle s'est sauvée du précipice qui menaçait de l'engloutir mais de cette façon elle s'est mise au rang des autres nations et sa Révolution a perdu l'intérêt général cosmopolite qui en avait fait auparavant la cause de tous les peuples» (p. 587). Il est donc clair que pour lui Bonaparte n'est pas le continuateur de la Révolution, mais l'homme auquel la France a 
remis son destin. Ce coup d'État n'est donc pas comparable aux autres, il signifie la fin et l'échec de l'expérience révolutionnaire. La France a voulu assurer son salut et il faut comprendre cet événement comme une autodéfense. Mais en même temps, le 18 Brumaire lui a fait perdre son caractère cosmopolite, elle est devenue un État comme les autres le citoyen du monde doit donc tirer les conséquences de cette nouvelle situation.

En particulier, Görres va envisager sous un angle nouveau, celui de l'intérêt national, le problème de la réunion de la rive gauche du Rhin. Il pense toujours que la Convention Nationale de Mayence avait eu raison de demander la réunion à la France, mais il se demande si, sept ans après, il est intelligent de la solliciter à nouveau. Il se pose trois questions la réunion est-elle toujours de l'intérêt des pays rhénans Le retour à ses anciens seigneurs serait-il néfaste N'y aurait-il pas une troisième solution

Pour répondre à la première question, il revient à la théorie des frontières naturelles et la met en cause au nom de la nature, lui opposant l'esprit national. Görres avait toujours été conscient de ce que le facteur linguistique et les caractères nationaux étaient des obstacles à la réunion, il l'écrit dans La paix universelle, un idéal ${ }^{6}$. Mais il avait pensé que ce n'étaient que des difficultés passagères et que l'éducation pourrait les éliminer. Maintenant, il découvre la profondeur des liens qui l'unissent au peuple allemand. Se situant dans la perspective de Herder, il déclare que «la langue est le grand lien qui unit les individus les uns aux autres » (p. 590). Il emprunte également à Herder l'idée que la langue et la culture d'un peuple sont étroitement liés et en conclut qu'on ne peut imposer à un peuple une nouvelle langue et une nouvelle culture « Là où un peuple conquérant imposait une nouvelle langue au peuple soumis, la culture marquait soudain le pas dans la progression qu'elle avait suivie jusque là » (p. 590). Lui qui avait pensé que l'élite rhénane pourrait jouer un rôle de médiateur entre les deux cultures pense maintenant que le heurt des deux langues donnera naissance à un idiome corrompu qui n'assurera qu'une compréhension insatisfaisante et entravera le développement culturel des Allemands. En revanche, ce qui peut unir les peuples, c'est l'humanité, mais l'idée d'humanité elle-même devient, dans sa nouvelle optique, dépendante de l'évolution des peuples « Il y a sans doute une chose qui est commune à tous les peuples sur toute l'étendue du globe, à savoir l'humanité mais cet élément commun, comme tout ce qui existe dans la nature n'est pas coulé en une masse compacte aux contours nets, il diminue ou augmente, monte ou descend, serpente en une ligne sinueuse et changeante à travers tous les peuples » (p. 590). Dans cette image aussi, l'influence de Herder est indéniable. Görres conclut ce développement en déclarant que c'est dans la nature morale de l'homme que la nature a indiqué les limites des États et que l'homme d'état doit en tenir compte et abandonner la théorie des frontières naturelles.

11 Görres brosse alors un tableau des caractères nationaux allemands et français qui n'a rien d'original, montrant qu'il y a un fossé entre eux, une véritable frontière naturelle. En revanche, les conclusions qu'il en tire sont tout à fait nouvelles sous sa plume « La langue et l'esprit national, les coutumes et les lois, dans la mesure où ces dernières dépendent des premiers et en procèdent, s'opposent donc puissamment à une union des deux peuples les vignes du Rhin et les oranges du midi ne poussent pas sous le même soleil la nature les a séparés et ce qu'elle veut savoir séparé ne se réunit point si aisément» (p. 593). La réunion lui apparaît maintenant comme une entreprise non seulement difficile, mais totalement artificielle. 

l'Italie lui paraît assez parlant. Après avoir étudié toutes les conséquences qui en résulteraient, il estime que les passions, la vengeance, la haine, les brutalités les attendraient si les princes reprenaient le pouvoir. Cette solution est donc totalement à rejeter.

recherche alors une troisième voie. Un conseiller d'État a proposé de faire de ce pays une " colonie de terre ». Mais cette solution n'a aucun des avantages de la réunion et garde tous ses inconvénients. Görres trouve même scandaleuse une telle proposition qui fut d'ailleurs aussitôt rejetée. Reste la mise en place d'une république indépendante, ce qui fut tenté lors des négociations au sujet de la République Cisrhénane. Pendant un court moment, le Directoire a encouragé cette création, puis a abandonné le projet au profit de la réunion. Cette solution est-elle encore possible après le 18 Brumaire? Görres pense qu'elle ne trouvera plus aucun écho favorable en France. En effet, le pays est riche et peut permettre à la France de retrouver son éclat. Celle-ci va donc tout mettre en œuvre pour le garder, ce pays lui permettant de retrouver les richesses perdues par la guerre. De leur côté, les princes allemands sont totalement opposés à cette solution qu'ils redoutent et feront tout pour l'empêcher. Il serait donc vain de vouloir reprendre ce plan bien qu'il soit le meilleur pour les Rhénans.

14 Ne restent donc plus que les deux premières solutions. Or, l'incorporation à la France présente encore quelques avantages la croissance de l'industrie, du mieux être de tous grâce à la réunion avec un peuple puissant, enfin la croissance de la culture puisque seront brisées les chaînes locales qui entravent son développement. En revanche, un retour à l'ordre féodal ne présenterait que des inconvénients. Mais c'est une chose que de vouloir la réunion et une autre que de la solliciter, surtout avant la conclusion du traité de paix. Pour les pays rhénans, cela ne présenterait que la garantie de leur avenir. Pour Bonaparte, il serait absurde de faire une démarche tendant à montrer qu'il veut garder les pays du Rhin alors qu'il songe à terminer la guerre, ou, si cela n'était pas possible, à en rejeter la responsabilité sur ses adversaires. L'attitude des autres puissances l'a obligé à choisir la guerre, ce n'est donc pas le moment de faire une démarche officielle pour demander la réunion. Görres et Eickemeyer rédigent alors une adresse qu'ils donneront quand le moment sera favorable. Dans cette adresse, ils décrivent les maux du passé, ceux du présent et demandent de l'aide pour l'avenir. Ils veulent être tranquillisés sur le sort futur du pays et plaident en faveur de la réunion qui, selon eux, « ravivra dans ce peuple son énergie et les sentiments de sa dignité en réunissant enfin tous les habitants sous l'étendart de la liberté devenus citoyens français, ils ne craindront plus la vengeance de leurs anciens maîtres» (p. 607). Pour mieux argumenter, ils vont jusqu'à déclarer que presque l'unanimité des concitoyens s'est déclarée pour cette réunion et en a déjà fait la demande à l'ancien Directoire, affirmation qui non seulement est fausse, mais contradictoire par rapport aux Résultats. Le Premier Consul leur affirme qu'ils peuvent compter sur l'amour de la justice du gouvernement français qui veillera toujours à leur bien.

15 En effet, en contradiction totale avec tout ce qu'il a dit jusque là et donc avec l'adresse envoyée aux Consuls de la République ${ }^{7}$, Görres affirme dans son texte que la grande majorité des Rhénans est hostile à la réunion. Celle-ci n'est d'ailleurs plus justifiée par l'idéal cosmopolite de la Révolution et il démontre qu'elle est antinaturelle. S'il la sollicite cependant dans l'adresse envoyée aux Consuls, c'est qu'elle représente un 
moindre mal par rapport à la réaction terrible que représenterait le retour du pays à ses anciens maîtres. Il admet que la France a réalisé d'importantes réformes grâce à la Révolution, nous l'avons vu, mais les cite sans grand enthousiasme et présente la réunion comme la moins mauvaise des solutions possibles, la seule pour échapper au pire. La meilleure solution eut été une république indépendante, mais elle n'a plus aucune chance d'être adoptée. Comme Görres avait demandé de pouvoir solliciter soit la réunion soit l'indépendance selon les circonstances, il opte en fin de compte sans enthousiasme pour la réunion. Par ailleurs, dans son texte, il donne une interprétation nouvelle du mouvement cisrhénan de 1797, associant l'idée de nationalité au problème de l'indépendance. La nature la plus spécifique de la patrie eut pu être préservée si elle avait été indépendante d'autre part, il présente pour la première fois ce projet comme une solution qui eut pu ménager la possibilité d'un futur rattachement à l'Allemagne. Or, cette idée n'a jamais effleuré les Cisrhénans et Görres projette ainsi manifestement ce qu'il pense en 1800 sur les événements de 1797. On la retrouve dans une lettre au baron de Stein du 4 août $1814{ }^{8}$ dans laquelle il présente le projet de république indépendante comme une parade à la réunion qui permettrait le rattachement ultérieur de la rive gauche du Rhin à l'Allemagne.

Dans l'ensemble, les théories de Görres sont très morales, comme l'est d'ailleurs la pensée politique de l'époque aussi bien en France qu'en Allemagne. Mais, contrairement à beaucoup de penseurs allemands et dans la ligne de Kant, il pense que les réformes politiques sont la condition du progrès moral. Jusqu'au 18 Brumaire, c'est de la gauche républicaine qu'il attend une action décisive contre la corruption et le pourrissement du régime. D'où son espoir au moment de prairial, car il est encore persuadé de la vocation cosmopolite de la Révolution. Son revirement après le 18 Brumaire s'explique par l'analyse de la situation qu'il fait aussitôt et l'interprétation nationale qu'il donne de l'événement d'une part et d'autre part son antipathie pour le peuple français qu'il découvre pour la première fois. Les caractères nationaux français et allemands lui paraissent incompatibles et cette découverte lui semble d'autant plus importante qu'il définit désormais l'État par les traits spécifiques d'un peuple, en particulier sa langue et sa culture.

Dans cette nouvelle perspective, il affirme la supériorité intellectuelle et morale de l'Allemand sur le Français et, pour la première fois, il parle de la grandeur du génie allemand. Resté fidèle à l'idéal révolutionnaire, il travaille pour un pays qui s'en éloigne de plus en plus. C'est parce qu'il pense que Bonaparte a mis fin à la Révolution le 18 Brumaire qu'il s'éloigne de la France dans l'espoir que l'Allemagne pourra devenir révolutionnaire, d'autant qu'il la juge moralement supérieure à la France. Toutefois, en mettant en avant l'idée d'une mission allemande, il reste progressiste, comme Fichte ou Hölderlin. Il veut toujours établir le règne de la liberté et son nationalisme reste conciliable avec le cosmopolitisme : puisque la France a failli, l'Allemagne doit prendre la relève pour guider les peuples, mais dans le même but que la France révolutionnaire. 


\section{NOTES}

1.Joseph VON GÖRRES, Das rothe Blatt, in Gesammelte Görres Werke, Köln 1929, vol. I.

2.Sur l'ambiguité de la politique du Directoire dans les pays rhénans et la réaction des révolutionnaires de ce pays, voir notre communication au colloque de Lille La perception de la Grande Nation en Rhénanie, 12-13 mars 1999.

3.Joseph VON GÖRRES, Der Rübezahl, ibid., vol. I, p. 540. En français dans le texte.

4.Joseph VON GÖRRES, Resultate meiner Sendung nach Paris im Brumaire des achten Jahres, ibid., vol.I, p. 554-608. Ici p. 555.

5. In W. SCHELLBERG, Joseph von Görres. Ausgewählte Werke und Briefe, 2. Band, Kempten 1911.

6.Joseph VON GÖRRES, Der allgemeine Friede, ein Ideal, in Gesammelte Görres Werke, Köln 1929, vol. I.

7.Il est vrai que celle-ci a été rédigée par Eickemeyer et que Görres s'est contenté de la signer.

8.M. BOTZENHART, Frh. von Stein, Briefe und sämtliche Schriften, Bd. 5, Stuttgart 1964, pp.

95-97.

\section{RÉSUMÉS}

Görres est un des rares penseurs révolutionnaires allemands à être passé à la pratique politique. Déçu par la politique extérieure du Directoire, il est partisan d'envoyer une députation à Paris afin de solliciter le rattachement à la France des territoires occupés. Il y arrive le 30 brumaire peu après le coup d'État. Contrairement à d'autres Allemands qui voient en Bonaparte le garant d'un retour à l'ordre, Görres redoute de "voir les conquêtes de la Révolution tout entières englouties par l'ambition d'un seul homme". Le 18 Brumaire lui parait la conséquence naturelle des événements qui l'ont précédé; le coup d'État signifie la fin et l'échec de la période révolutionnaire et Görres va envisager sous un angle nouveau le problème de la réunion de la rive gauche du Rhin présentée seulement comme un moindre mal. Les caractères nationaux français et allemands lui paraissent désormais incompatibles. Il met en avant l'idée d'une mission allemande: c'est à ce pays de prendre la relève pour guider les peuples, car il est moralement supérieur.

A German View of 18 Brumaire: the "Results of my Mission to Paris in Brumaire Year VIII" by Joseph von Görres. Görres is one of the few German revolutionary thinkers to have actually been involved in politics. Disapppointed by the foreign policy of the Directory, he was in favour of sending a delegation to Paris to demand the union of the occupied lands with France. He arrived there on 30 Brumaire, shortly after the coup d'état. Unlike many Germans who saw in Bonaparte the safeguard to a return to order, Görres feared to "see the achievements of the Revolution entirely swallowed up by the ambition of a single man". The coup of 18 Brumaire appeared to him the natural consequence of the events which preceded it: both the end and the failure of the Revolution. Thus, Görres adopted a different stance on the issue of the annexation 
of the Left Bank of the Rhine, seeing it as the lesser of two evils. The national characters of France and Germany now seemed irreconcilable. He floated the idea of a new calling for Germany, whose moral superiority empowered her to replace France as a guide to other nations.

Görres è uno dei rari pensatori rivoluzionari tedeschi passato alla pratica politica. Deluso dalla politica estera del Direttorio, egli spinge per inviare una delegazione a parigi, allo scopo di sollecitare l'annessione alla Francia dei territori occupati. Egli vi arriva il 30 Brumaio, poco dopo il colpo di Stato. Contrariamente ad altri tedeschi, che vedono in Bonaparte il garante di un ritorno all'ordine, Görres teme di "vedere le conquiste della Rivoluzione interamente fagocitate dalle ambizioni di un solo uomo". Il 18 Brumaio gli sembra la conseguenza naturale degli eventi che l'hanno preceduto; il colpo di Stato significa la fine e l'insuccesso del periodo rivoluzionario e Görres si appresta a considerare sotto una visuale nuova il problema della riunione della riva sinistra del Reno, presentata soltanto come un male minore. Il carattere nazionale francese e tedesco gli appaiono ormai incompatibili. Egli porta avnti l'idea di una missione tedesca: spetta a questo paese sostituirsi nella guida dei popoli, in quanto moralmente superiore.

Görres es uno de los raros pensadores revolucionarios germanos que pasó a la práctica política. Decepcionado por la política exterior del Directorio, es partidorio del envio de una delegación en Paris para solicitar la incorporación de los territorios a Francia. Consíguelo el 30 de brumaire, un poco despues del golpe de estado. A la diferencia de otros germanos que ven en Bonaparte el garante de la vuelta a la orden, Görres teme de "ver las conquistas de la revolución enterradas por la ambición de un solo hombre". Analiza el 18 Brumaire como la consecuencia natural de los acontecimientos anteriores; el golpe de estado significa el fracaso del periodo revolucionario y Görres ve de otra manera el problema de la reunión de la orilla e izquierda del Rin, considerada solamente como un mal menor. Los caracteres nacionales francos o germanos le parecen ya incompatibles. Habla de una misión germana: por su superioridad moral este país tiene que tomar el relevo para guiar a los pueblos.

Görres ist einer der seltenen deutschen Revolutionäre, die eine politische Tätigkeit hatten. Er wurde von der äußeren Politik des Direktoriums sehr enttäuscht und wollte eine Delegation nach Paris senden, um den Anschluß an Frankreich der besetzten Länder zu fordern. Er kommt am 30. Brumaire an, kurz nach dem Staatsstreich. Im Gegenteil zu vielen Deutschen, die in Bonaparte den Bürgen einer Rückkehr zur Ordnung sehen, befürchtet Görres "die Ausbeute der ganzen Revolution von dem Ehrgeize eines Einzigen verschlungen zu sehen". Der 18. Brumaire erscheint ihm als die natürliche Folge der Ereignisse, die ihm vorangegangen sind; dieser Staatsstreich bedeutet zugleich das Ende und das Scheitern der Revolution. Deshalb sieht er die Frage des Anschlusses des linken Rheinufers mit anderen Augen und diese Lösung wird nur noch als das kleinere Übel dargestellt. Die französischen und deutschen Nationalcharaktere scheinen ihm jetzt unvereinbar. Er hebt die Idee einer deutschen Mission hervor: Deutschland als moralisch höheres Land soll nun an Stelle Frankreichs die Völker leiten.

\section{AUTEUR}

\section{MARITA GILLI}

Université de Franche-Comté 